\title{
Rapid Evidence Assessment Protocol for the the Meta-analysis of Initiatives, Interventions and Programmes that Target Rural NEETs
}

\author{
Paul Flynn ( $\sim$ paul.flynn@nuigalway.ie) \\ National University of Ireland Galway https://orcid.org/0000-0002-0957-8605 \\ Veronica McCauley \\ National University of Ireland Galway \\ Alen Mujcinovic \\ University of Sarajevo \\ Vesela Radovic \\ University of Belgrade \\ Stefan Bojnec \\ University of Primorska

\section{Francisco Simoes} \\ ISCTE-Instituto Universitário de Lisboa: ISCTE-Instituto Universitario de Lisboa
}

\section{Protocol}

Keywords: Rapid Evidence Assessment, Protocol, Rural, NEETs

Posted Date: March 26th, 2021

DOI: https://doi.org/10.21203/rs.3.rs-337475/v1

License: () (1) This work is licensed under a Creative Commons Attribution 4.0 International License. Read Full License 


\section{Abstract}

Background The dependency of rural economies on agricultural practices for survival has been in decline for a number of years, seriously threatening rural communities sustainability. It is now evident that attracting young people into agricultural professions is proving difficult. As a consequence, policy measures that aim to enhance the likelihood of rural employment for young people or rural NEETs have been introduced in countries with the aim of reducing at-at-risk-poverty rates and supporting the long term viability of rural economies. The aim of this rapid review is to summarise the published literature reporting on the effectiveness of initiatives, interventions and programmes that target rural NEETs.

Methods The following databases will be searched: Web of Science, SCOPUS, EBSCOhost, ERIC and ProQuest. Internet searching through Google Scholar will also be carried out. The result of the search will be downloaded and saved to Mendeley. Informed by PRISMA guidelines, duplicates will be removed. The titles and abstracts of all remaining studies will be screened by at least two independent reviewers informed by the study inclusion and exclusion criteria. Where a decision to include or exclude cannot be reached by the two independent reviewers an additional reviewer will adjudicate. All results, screening process results will be documented in tabular form and communicated in a PRISMA flow diagram.

Discussion This rapid review protocol will provide a time limited state-of-the-art evidence report, specific to the challenges experienced by rural NEETs, that will help those involved in policy development, policy response initiatives and proximal implementation. It is intended that additional opportunities for innovation and/or research may be communicated to relevant stakeholders. Hence, they will be able to create a useful framework for embedding rural youth in the context of so needed structural transformation of rural communities.

Systematic Review Registration This protocol has been submitted to the International Prospective Register for Systematic Reviews - PROSPERO, published, and assigned the following reference number: CRD42021236794.

\section{Background}

The acronym NEET refers to youths aged between 15 and 34 years old who are excluded from employment, education and Training (Eurostat 2020). However, historically, the NEET (Not in Employment, Education and Training) demographic have been depicted as a largely homogenous group (Furlong 2006). While in recent years more attention has been paid to the subtleties of in-group variability for this demographic, it has taken some time for reexaminations of the definition of NEETs to acknowledge a need to redefine the age range and resulted in a change form 16-18 years to 15-34 years. In addition to this a broadening of the inclusion criteria across the newly defined age range has emerged with sub-classifications that focus on temporal, and contextual conditions (Mascherini 2019).

Although several authors (Furlong 2006; Mascherini 2019) have extensively addressed the need to pay attention to NEETs different trajectories and profiles, geographic criteria, namely origin, have been kept 
outside this discussion. In this context, scholars' indifference towards rural NEETs is puzzling, especially when taking into account international figures. In 2019, $13.6 \%$ of youths in EU28 aged 15-34 were NEETs. NEETs' distribution seems balanced across EU rural areas (14.7\%) suburbs (14.1\%) and cities (12.8\%). Still, disparities across countries tell a very different story, showing a clear North/South divide on this matter. In Romania (21.7\%), Italy (24.8\%), Greece (25.6\%) or Bulgaria (28.6\%), one out of four or one out of five youths living in the countryside are NEETs. These figures contrast with very low rural NEETs rates in countries such as The Netherlands (5.6\%), Austria (6.6\%) or Sweden $(7.2 \%)$. Thus, the sustainability of rural communities is pretty much at stake in countries where overall institutional support for young people is shrinking, less recurrent or inefficient (Bello and Cruzzocrea 2018; Walther 2006), notably in rural areas (Simões and Rio 2020). This challenge is even more relevant considering that rural NEETs, as well as other groups of disadvantaged youths are the largest share of those remaining in the countryside (Farrugia 2016). These youths are more affected by involuntary immobility (Carling and Schewel 2018), meaning that they are reluctant to stay in rural areas, but do not have the resources to leave. So, in short, their futures depend on the available opportunities. Matching these youths needs for decent jobs with local resources, namely

\section{Rural NEETs and the agricultural sector: Matching needs and resources}

Against this backdrop and given the dependency of rural economies on agricultural practices for survival has been in decline for a number of years, seriously threatening rural communities sustainability. It is now evident that attracting young people into agricultural professions is proving difficult. As a consequence, policy measures that aim to enhance the likelihood of rural employment for young people have been introduced in countries with the aim of reducing at-at-risk-poverty rates and supporting the long term viability of rural economies (EC, 2020a, 2020b). This action is informed, in part, by the prevailing role of higher added value non-agricultural activities in planning sustainable living in rural areas where labour demands are disparate and dependent on a vibrant and socially interconnected rural ecosystem. Understanding these activities and the impact that they have is important if a more concerted multi-level response to the challenges of rural NEETs is to be possible.

In theory, policies seem appropriate to improve rural NEETs' involvement in the sector across European countries which can lead into a kind of voluntary immobility, meaning a willingness from these young people to stay and strive in rural areas. However, in practical terms, policy packages continue to fail to match the agriculture sector needs for human capital with employment needs of rural NEETs. This mismatch can be ascribed to a series of interrelated factors, with most of them remaining unaddressed by decision-making. One of them is the excessive protectionism of the sector that prevents newcomers with limited background knowledge of the sector such as NEETs of finding a job in the sector (Greer 2017). Aside, it is well documented the inability and lack of resources of the local employment services to motivate these youths for the activities in the agriculture sector (Shore and Tosun 2019). These limitations of employment services overlap formal educational systems that overemphasise labor market demands, to the detriment of community opportunities in the agriculture sector, meaning that local resources are not under the radar of potential professional choices promoted by school and training 
centres (Simões 2018). Moreover, families resist the idea of seeing their children involved in agriculture, experiencing that possibility as a kind of failure in providing them a bright and successful future (Simões 2018). At the individual level, all these barriers lead to the formation of negative stereotypes about the sector among youths as a mainly a male-dominated professional area involving low wages and dirty, undifferentiated work (Simões 2018; Simões and Brito do Rio 2020). However, when having the opportunity to be involved in training and agriculture activities, NEETs acknowledge that these images are oversimplifications of the real complexity that agriculture activities encompass (Simões and Brito do Rio 2020).

Rural labour markets are structurally and distance specific and there are significant challenges associated with supporting such action. They provide narrower and limited job offers for potential career development, often for relatively lower-skilled jobs in less proximity spatial locations. Supporting cooperation among the various actors within the rural labour setting, or rural ecosystem, is challenging. The macro level stakeholders include: the labour market; the education system; and local employment development initiatives, which face significant challenges in relation to removing labour market mismatches at rural and regional levels as employment and/or unemployment as a factor of social exclusion among youth can be more severe in rural areas. This can be particularly challenging when weak family support is linked with weak formal support systems in the society creating mismatches between the education system and labour market integration. At the micro-level, for rural youth, who fall into the NEET category and where the absence of opportunity in the rural context is persistent they can often go unnoticed or hidden from view. While these rural NEETs can present as registered unemployed and also within the reporting statistics of various different state funded initiatives, interventions and programmes, in the case of Rural NEETs, there is a dearth of reporting categories that highlight the specificity of this group resulting in their presence being largely overlooked within official dissemination.

In light of the above, the overall aim of this rapid review is to identify initiatives, interventions, and programmes that seek to address the specific challenges experienced by rural NEETs by asking the following review question: How do initiatives, interventions or programmes, which aim to reestablish participation in education, employment and training for young people aged between 15 and 34 years who experience marginalisation as a consequence of rural dwelling? The overall aim of this review is to identify, synthesise and consider evidence of the impact of initiatives, interventions or programmes that specifically target the reengagement in education, employment and training of rural young people aged between 15 and 34 years. To achieve this the following specific actions will be taken:

- A systematic review will be carried out with a view to reviewing, synthesising and evaluating through a quality appraisal process experimental evidence on the effects of initiatives, interventions or programmes on rural NEETs.

- Estimation of the impact of initiatives, interventions or programmes on rural NEETs' relationship with education, employment and training.

- Identification of key characteristics of initiatives, interventions or programmes mitigate rural isolation and marginalisation. Of particular interest are features of initiatives, interventions or programmes 
that impact on NEETs' holistic wellbeing as a consequence of re-engagement with education, employment and training.

- Additional, emergent, impacts of reviewed initiatives, interventions or programmes targeted at rural NEET reengagement with education, employment and training will also be investigated resulting in the identification of emergent gaps and needs for future research areas will also emerge.

\section{Methods/design}

\section{Protocol and Registration}

This rapid evidence assessment is informed by the World Health Organisation (WHO) (2017) and UK Department for Environment, Food \& Rural Affairs (DEFRA) (2015) publications that outline best practice for the development or Rapid Evidence Assessments. This protocol has been submitted to the International Prospective Register for Systematic Reviews - PROSPERO. Currently there is no standardised reporting process for rapid reviews that are carried out. Consequently, this protocol has adapted the PRISMA-P and associated checklist (Additional File 2) as a framework to report on the findings of the review.

\section{Inclusion \& Exclusion Criteria}

The research team are interested in identifying, synthesising and considering evidence of the impact of initiatives, interventions or programmes that specifically target the reengagement of rural NEETs in three areas: education, employment and training. The research team is primarily interested in the impact of initiatives, interventions or programmes on rural NEETs' relationship (in sum or in part) with education, employment and training. Studies that are qualitative, quantitative or mixed methods will be included. Studies that do not include imperial data will not be included in this review. Following on from this, in order for publications and reports to be considered eligible for inclusion in this review the following inclusion and exclusion criteria, following on from the formation of the aforementioned research question using the PICO elements are useful: population, intervention, comparison, and outcomes. Therefore, PICO model is applied:

\section{Population}

The review will focus on young people aged between 15 and 34 years who live in rural areas and who are not in education, employment and training at the time of the studies being carried out. Where studies include participants that fall outside of the above age range, if the mean age range of the study falls between 15-34 years the study will be included. Where studies do not focus exclusively on rural NEETs, where the datasets presented by the study are not presented separately and where the authors cannot provide additional information the study will be excluded.

\section{Intervention}


Interventions will be considered if they adhere to the target population parameters. Studies that focus on formal, non-formal, informal, place-based learning, community engagement, outreach activities, media programmes, sports initiatives, enterprise interventions, career pathway programmes and general educational initiatives, interventions and programmes. In addition studies that incorporate combinations of the above will also be included.

\section{Comparison}

While it is unlikely that studies with concurrent control or comparison groups will emerge in the review process, if they are present they will be included. If sub-group analysis is possible this will be explored specific to the impact of initiatives, interventions and programmes on NEETs' relationships with education, employment and training.

\section{Outcomes}

The impact of initiatives, interventions and programmes on NEETs' relationships with education in terms of re-engagement and/or progression with learning or employment outcomes will be included as will studies that report on the impact of initiatives, interventions and programmes on holistic wellbeing outcomes.

\section{Search Strategy}

As part of an initial investigation the research team carried out an initial search for extant systematic reviews and meta-analysis. This initial search revealed a dearth of reviews and meta-analysis focusing on initiatives, interventions or programmes targeted at rural NEET reengagement with education, employment and training. This systematic review will employ a standardised search strategy using defined terms within search strings (Additional File 1). Databases deemed most likely to return a high number of relevant results will be searched. It is acknowledged by the research team that additional searches may be warranted. The following databases will be searched: Web of Science, SCOPUS, EBSCOhost, ERIC and ProQuest. Internet searching through Google Scholar will be carried out as well as forward and backward tracking of citations from studies that are included in the review. Hand searches of journals will be carried out if warranted. Where necessary additional follow up contact with authors, experts and research groups will further inform this review. The review will only include peer reviewed studies published in English, however, if a study published in an alternate language is deemed relevant it will be included. Consequently no grey literature will be included in this review. The result of the search will be downloaded and saved to Mendeley. Informed by PRISMA guidelines, duplicates will be removed. The titles and abstracts of all remaining studies will be screened by at least two independent reviewers informed by the study inclusion and exclusion criteria. Where a decision to include or exclude cannot be reached by the two independent reviewers an additional reviewer will adjudicate. All results, screening process results will be documented in tabular form and communicated in a PRISMA flow diagram.

\section{Data Extraction and Quality Appraisal}


After the final research returns have been collated two independent reviewers will read all titles and abstracts after duplicates have been removed. The titles and abstracts will be screened in accordance with inclusion and exclusion criteria. Where it is not possible to determine relevance from the title and abstract alone, at this stage the full paper will be accessed to determine whether it should be included. Where there is disagreement between reviewers a third independent reviewer will review the paper and attempts will be made to resolve the disagreement through discussion. If at this point there is still disagreement the paper will be retained for future reference.

The next step in this process will be full paper screening. Where a paper is deemed not to meet the review inclusion criteria it will be rejected following the dual appraisal method as described above. Papers that meet the inclusion criteria will have the following data extracted and highlighted in table format with brief descriptions where appropriate:

- Authorship

- Year of publication will be listed. This will be a time limited study of 10 years.

- Aims of the study

- Sampling strategy and characteristics (also process of recruitment, drop out)

- Methodology

- Description of the interventions

- Key findings/outcomes

- Overall quality rating of the study using the CASP Appraisal Tool Checklist (2018).

\section{Data Analysis and Synthesis}

It is anticipated by the research team that a wide range of study designs and outcomes will be returned as part of this review. The data extracted from this review will be presented in a thematic narrative format. It is hoped that this will include graphics and/or evidence tables. Where the review team determines that the best course of action is to use statistical summaries this approach will be implemented however, it is the view of the review team, informed by domain expertise, that this is unlikely.

\section{Assessing the Risk of Bias}

Initially, each study will be reviewed by two independent reviewers for quality using the CASP Appraisal Tool Checklist (2018). The intention of the review is not to exclude studies on the basis of bias rather it is to note the quality of the studies being reviewed. To examine for selective reporting of results, papers will be examined for indicators of multiple use of samples included in the papers. For example, this may include indicators that data has been drawn from larger projects, a published thesis or other papers. Papers that are determined to fall under this category will be checked against alternate sources, where available, to outcomes relevant to this review are being presented. Finally, GRADE-CERQual guidelines will also be followed in the assessment of outcome quality for methodological limitations, coherence, adequacy of data and relevance. 


\section{Amendments to the Protocol}

Where there are unavoidable significant deviations from this protocol, such amendments will be recorded and reported within the result of the review.

\section{Discussion}

This rapid review protocol will identify, synthesise and consider evidence of the impact of initiatives, interventions or programmes that specifically target the reengagement in education, employment and training of rural young people aged between 15 and 34 years. It will achieve this by providing a time limited state-of-the-art evidence report, specific to the challenges experienced by rural NEETs, that will help those involved in policy development, policy response initiatives and proximal implementation. It is intended that, depending on the outcome of the review process, additional opportunities for innovation and/or research may be communicated to relevant stakeholders. Hence, they will be able to create a useful framework for embedding rural youth in the context of so needed structural transformation of rural communities.

\section{List Of Abbreviations}

NEET: Not in Employment, Education and Training

PICO: Population, intervention, comparison, outcomes.

PRISMA-P: Preferred Reporting Items for Systematic Reviews and Meta Analyses

REA: Rapid Evidence Assessment

\section{Declarations}

\section{Ethics Approval and Consent to Participate}

Not applicable

\section{Consent for Publication}

Not applicable

\section{Availability of data and materials}

Data sharing not applicable to this article as no datasets were generated or analysed during the current study

\section{Competing interests}


The authors declare that they have no competing interests

\section{Funding}

This work was supported, in part, by the Ministry of Education, Science and Technological Development of the Republic of Serbia (Contract No. 451-03-68/2020-14/200053).

\section{Authors' Contributions}

$\mathrm{PF}$, is the principal investigator of this review and contributed to review conception, refinement of the design and writing the manuscript. VMcC, AM, VR contributed to review conception, refinement of the design and writing the manuscript. PF, VMcC, AM, VR, SB \& FS collaboratively drafted the manuscript. All authors were involved in the conception, design and iterative refinement of the final manuscript.

\section{Acknowledgements}

The authors would like to acknowledge the support of their individual affiliate institutions regarding the development of this research project and the support of COST Action CA18213 Rural NEET Youth Network, supported by COST (European Cooperation in Science and Technology).

In addition, the authors would like to acknowledge the Ministry of Education, Science and Technological Development of the Republic of Serbia (Contract No. 451-03-68/2020-14/200053).

\section{Authors' Contributions}

$\mathrm{PF}$, is the principal investigator of this review and contributed to review conception, refinement of the design and writing the manuscript. VMcC, AM, VR contributed to review conception, refinement of the design and writing the manuscript. PF, VMcC, AM, VR, SB \& FS collaboratively drafted the manuscript. All authors were involved in the conception, design and iterative refinement of the final manuscript.

\section{Authors Information}

Not applicable

\section{References}

Carling J, Schewel K. Revisiting aspiration and ability in international migration. Journal of Ethnic and Migration Studies. 2018 Apr 26;44(6):945-63.

Collins A, Coughlin D, Miller J, Kirk S. The production of quick scoping reviews and rapid evidence assessments: A how to guide; 2015.

Cuzzocrea V, Bello BG. Introducing the need to study young people in Italy. In Journal of Modern Italian Studies; 2018. 
European Commission. Living conditions in Europe - poverty and social exclusion. Brussels: Office for Official Publications of the European Commission; 2020a.

European Commission. Young farmers. Brussels: European Commission Brussels: Office for Official Publications of the European Commission; 2020b.

Eurostat. Statistics on young people neither in employment nor in education or training: Statistics explained; 2020. https://ec.europa.eu/eurostat/statistics-explained/index.php

Farrugia D. The mobility imperative for rural youth: the structural, symbolic and non-representational dimensions rural youth mobilities. Journal of Youth Studies. 2016 Jul 2;19(6):836-51.

Furlong A. Not a very NEET solution: representing problematic labour market transitions among early school-leavers. Work, employment and society. 2006 Sep;20(3):553-69.

Greer A. Post-exceptional politics in agriculture: an examination of the 2013 CAP reform. Journal of European Public Policy. 2017 Nov 29;24(11):1585-603.

Mascherini M. Origins and future of the concept of NEETs in the European policy agenda. InYouth Labor in Transition 2019 (pp. 503-529). Oxford University Press.

Shore J, Tosun J. Assessing youth labour market services: Young people's perceptions and evaluations of service delivery in Germany. Public Policy and Administration. 2019 Jan;34(1):22-41.

Simões F. How to involve rural NEET youths in agriculture? Highlights of an untold story. Community Development. 2018 Oct 20;49(5):556-73.

Simões F, do Rio NB. How to increase rural NEETs professional involvement in agriculture? The roles of youth representations and vocational training packages improvement. Journal of Rural Studies. $2020 \mathrm{Apr}$ 1;75:9-19.

Tricco AC, Langlois E, Straus SE, World Health Organization. Rapid reviews to strengthen health policy and systems: a practical guide. World Health Organization; 2017.

Walther A. Regimes of youth transitions: Choice, flexibility and security in young people's experiences across different European contexts. Young. 2006 May;14(2):119-39.

\section{Supplementary Files}

This is a list of supplementary files associated with this preprint. Click to download.

- NEETREAPROTOCOLADDITIONALFILE1.pdf

- NEETREAPROTOCOLPRISMAP2015CHECKLISTADDIONALFILE2.pdf 